\title{
Correction to: Of Mink and Men? Surveilling Human Attitudes at the Zoonotic Human-Wildlife Boundary
}

\author{
Meredith L. Gore(1), ${ }^{1}$ Jessica B. Rizzolo, ${ }^{2}$ and Gary J. Roloff ${ }^{2}$ \\ ${ }^{1}$ Department of Geographical Sciences, University of Maryland, 7251 Priekart Drive, College Park, MD 20741 \\ ${ }^{2}$ Department of Fisheries and Wildlife, Michigan State University, 480 Wilson Road, East Lansing, MI 48823
}

Correction to: EcoHealth

HTTPS://DOI.ORG/10.1007/s10393-021-01548-6

In the original version of this article, the family name of Jessica B. Rizollo was incorrectly written. The correct name should be Jessica B. Rizzolo.

The original article has been corrected. 